\title{
Mitohormesis in exercise training
}

Troy L. Merry ${ }^{1 \dagger}$ and Michael Ristow ${ }^{1}$

${ }^{1}$ Energy Metabolism Laboratory, Swiss Federal Institute of Technology (ETH), 8603 Zurich, Switzerland

${ }^{\dagger}$ To whom correspondence should be addressed:

\author{
Troy Merry
}

ETH Zürich

Energy Metabolism Laboratory

Schorenstrasse 16, 8603 Schwerzenbach, Zurich, Switzerland

Tel +4144655 7223

Fax +4144655 7206

Email: troy.merry@gmail.com

Keywords: mitochondria, reactive oxygen species, ROS, hormesis, exercise, skeletal muscle 


\begin{abstract}
Hormesis is a process whereby exposure to a low dose of a potentially harmful stressor promotes adaptive changes to the cell that enables it to better tolerate subsequent stress. In recent years this concept has been applied specifically to the mitochondria (mitohormesis), suggesting that in response to a perturbation the mitochondria can initiate and transduce a signal to the nucleus that coordinates a transcriptional response resulting in both mitochondrial and non-mitochondrial adaptations that return and maintain cellular homeostasis. In this review we summarize the evidence that mitohormesis is a significant adaptive-response signaling pathway, and suggest that it plays a role in mediating exerciseinduced adaptations. We discuss potential mitochondrial emitters of retrograde signals that may activate known exercise-sensitive transcription factors to modulate transcription responses to exercise, and draw on evidence from mitochondrial dysfunction animal models to support a role for mitohormesis in mitochondrial biogenesis. Studies directly linking mitohormesis to the exercise training response are lacking, however mounting evidence suggests numerous signals are emitted from the mitochondria during exercise and have the potential to induce a nuclear transcription response, with reactive oxygen species (ROS) being the primary candidate.
\end{abstract}


Abbreviations: AMPK, 5' adenosine monophosphate-activated protein kinase; CaMK, $\mathrm{Ca}^{2+1}$ calmodulin-dependent; CIT2, citrate (Si)-synthase; clk-1, clock-1; COQ7, Coenzyme Q7 homolog, ubiquinone; COX, cytochrome oxidase; ETC, electron transport chain; GLUT, glucose transporter; HIF-1, hypoxia inducible factor; JNK, c-Jun N-terminal kinases; HSF, heat shock factor; HSP, heat shock protein; MAPK, mitogen-activated protein kinases; Mckl, murine clock; mTOR, mechanistic/mammalian target of rapamycin; mtTFA, mitochondrial transcription factor A; mtDNA, mitochondrial DNA; MOTS-c, mitochondrial open reading frame of the 12S rRNA-c; NFE212, nuclear factor erythroid 2-related factor 2; NF- $\mathrm{BB}$, nuclear factor kappa-light-chain-enhancer of activated B cells; NNMT, Nicotinamide NMethyltransferase; $\mathrm{PGC} 1 \alpha$, peroxisome proliferator-activated receptor gamma coactivator $1 \alpha$; ROS, reactive oxygen species; Rtg, Retrograde regulation protein; RXR $\alpha$, retinoid X receptor alpha; UPR ${ }^{\mathrm{mt}}$, mitochondrial unfolded protein response. 


\section{Hormesis and exercise}

Exercise training involves the repeated exposure to an acute increase in metabolic, thermoregulatory, hypoxic, oxidative and mechanical stress. It is this exposure that stimulates compensatory physiological adaptations that improve tolerance to subsequent similar stressors [1]. Achieving such adaptation is the purpose of undertaking regular exercise, and underpins the protective nature of exercise against the development of various chronic diseases [2]. The process by which an organism adapts to a specific stress in an effort to return the body to normal homeostasis was initially termed 'general adaptation syndrome' [3], before being broadened to what we now refer to as the hormesis theory $[4,5]$. The premises of this theory is that exposure to low continuous or higher intermittent sub-lethal doses of a stressor, that would otherwise be harmful at larger or chronic doses, promotes favorable biological adaptations which protect against greater subsequent stress. Hormesis diverges from the traditional liner response, where increasing exposure to potentially harmful stressors results in increasing levels of damage, and is best represented by a J- or bell-shaped curve indicating that very low exposure or very high exposure is detrimental, while moderate exposure is advantageous or even necessary (Figure 1).

The principle of hormesis dates back to the 16th century when Paracelsus stated sola dosis facit venenum, i.e. only the dose would make the poison [6]. The term hormesis was coined later following the observation that very dilute doses of poisonous substances stimulate the growth of plants or yeast $[7,8]$, and appears to apply directly to the exercise training response [9]. For example, endurance exercise depletes muscle glycogen and increases core temperature, and in response to training muscle glycogen storage are increased and thermoregulation is improved. If these stressors are further elevated by initiating exercise in a glycogen depleted state or training in the heat, exercise training induced improvements in muscle glycogen storage [10] and thermoregulation [11] are further enhanced. Conversely, 
extreme exposure to exercise heat-stress can lead to heat exhaustion [11], and chronic depletion of muscle glycogen through excessive exercise training contributes to central and peripheral fatigue associated with overtraining syndrome [12]. Therefore, and in line with the hormesis concept, increasing exercise stress can enhance cellular adaptations to an extent; however, once a threshold level of stress is exceeded exercise stress can have detrimental affects on cellular function.

\section{Mitohormesis}

In recent years the hormesis theory has been extended specifically to the mitochondria (mitohormesis) [13-16], with the concept being that mild perturbations in mitochondrial homeostasis coordinate a nuclear and cytosolic response that leaves the whole cell less susceptible to future perturbation. Such responses are not limited to acute cytoprotective mechanisms but can induce long-term metabolic alterations and stress resistance. During exercise mitochondria metabolism in increased to meet the energy demands of the exercise task. Therefore, if the mitochondria are capable of regulating their own, as well as cell-wide and possibly system-wide, responses to changes in homeostasis it stands to reason that mitohormesis signaling would be a central mechanism regulating exercise-mediated adaptation. In this article we will review the evidence that mitohormesis is contributing to the signaling of exercise adaptation, and postulate potential mediators of the mitohormetic response.

\section{Mitochondrial retrograde signaling}

The mitochondrial genome encodes for only a handful of proteins associated with the oxidative phosphorylation system, with the majority of mitochondrial proteins being encoded in the nucleus [17]. In order for the cell to respond to changes sensed by the mitochondria, signals must be transduced in a reverse or 'retrograde' direction from the mitochondria to the 
cytoplasm and nucleus (Figure 2). The majority of work to identify mitochondrial retrograde signals has come from budding yeast models (Saccharomyces cerevisiae), where mitochondrial DNA (mtDNA) can be removed or mutated without dramatically affecting the normal function of the cell. Early studies showed that removal of mtDNA alters the genomewide response to comprised mitochondria in a perturbation-specific manner, effectively establishing the existence of a signal transduction pathway between the mitochondria and nucleus [18-20]. The CIT2 gene was then observed to be the major nuclear target of mitochondrial perturbation in yeast [21], and this allowed for identification of retrograde regulatory proteins $(\mathrm{Rtg}) 1,2$ and 3 as transcription factors targeting CIT2 [22, 23]. In yeast Rtg2 appears to be the upstream sensor for mitochondrial perturbations and acts via Rtg1 and $\operatorname{Rtg} 3$ to coordinate a nuclear response [20, 24]. Importantly, Rtg2 activity is regulated by metabolites such as glutamate and ammonium [19], and is susceptible to inhibition by the nutrient-sensing kinases TOR (target of rapamycin) [25], thus providing a potential mechanism whereby changes in the mitochondria can affect cell-wide responses.

The Rtgs are now generally accepted to be the major transducers of retrograde (and potentially mitohormetic) signals in yeast, however, identifying candidates in mammalian cells has proven to be more complex. Through the varied manipulation of mtDNA content in cells, Chae et al. [26] has recently identified more than 70 transcription factors that are potentially involved in mitochondrial retrograde signaling. Primary candidates include those transcriptions factors that are also responsive to perturbations in mitochondrial respiration and include peroxisome proliferator-activated receptor gamma, coactivator 1 alpha (PGC1 1 ), hypoxia inducible factor (HIF-1), mitochondrial transcription factor A (mtTFA), nuclear factor erythroid 2-like 2 (NFE2L2), nuclear factor kappa-light-chain-enhancer of activated B cells $(\mathrm{NF}-\kappa \mathrm{B})$, c-Jun N-terminal kinases $(\mathrm{JNK})$ and retinoid X receptor alpha $(\mathrm{RXR} \alpha)[15,24$, 
26]. It is likely that specific transcription factors may be targeted dependent on type/extent of perturbation applied to the mitochondria to coordinate a perturbation specific response.

Importantly, potential mammalian mitohormetic signal transducers include the main transcriptional candidates mediating metabolic adaptation to exercise training, with PGC1 $\alpha$ being implicated in regulating numerous exercise adaptation processes but primarily mitochondrial biogenesis, mTOR is involved in resistance-induced hypertrophy, and NF- $\mathrm{kB}$ as a coordinator of the antioxidant response to exercise [27-29]. In order to facilitate nuclear and cytoplasmic response to mitochondrial stress, the mitochondria must emit a signal to activate these potential mitohormesis transcription factors (Figure 2). In the next section potential mitochondrial stress signal emitters will be discussed in the context of exercise.

\section{Potential mitochondrial retrograde signal emitters and exercise}

\section{Reactive oxygen species (ROS)}

One of the most well recognized mitochondrial emitters that act as the proximal signaling for mitochondrial controlled cell- and potentially system-wide adaptation are $\operatorname{ROS}[14,15]$. Mitochondria produce ROS as a normal by-product of oxidative phosphorylation. An increase in ROS production as a result of increased metabolic flux or mitochondrial dysfunction has been implicated in the progression of diseases such as atherosclerosis, dementia, diabetes and cancer, and associated with the aging process [30-34]. Consequently, a number of studies have suggested that lowering oxidative stress prolongs life expectancy [35-39], however this is not a consistent finding [16, 40,41]. In humans and animals where physiological levels of ROS do not promote chronic disease [42], interventions that lower ROS levels (i.e. antioxidant supplementation) do not reduce mortality rates [43-49] and may actually promote the development of disease [50-56]. Indeed, physical exercise training substantially increases ROS systemically and in skeletal muscle [57], yet reduces the incidents of disease and extends 
life expectancy [2]. Consistent with these observations ROS are increasingly being recognized as essential signaling molecules for normal homeostatic functions including insulin and growth factor signaling, vascular tone regulation and immune responses [58-61].

Lifespan-extending interventions such as calorie restriction and disruption of the insulin/IGF1-signaling pathway increase mitochondrial respiration and confer enhancements in antioxidant and cryoprotective systems [16, 41, 62-64]. In C. elegans, these lifespanextending interventions have been shown to require an acute increase in mitochondrialderived ROS production in order to up regulate cellular stress defense systems and ultimately extend lifespan [16, 41]. This strongly suggests that mitochondrial ROS is an important mitohormetic emitting signal, but also questions why a ROS-mediated increase in antioxidant capacity promotes longevity and general antioxidant supplementation does not. This is probably the result of the response to oxidative stress not being limited to the antioxidant system but also up-regulating other cryoprotective and phase II detoxifying enzymes. In addition, a complex network of compartmentalized ROS eliminating systems regulates endogenous oxidative balance, and generalized antioxidant supplementation is likely to upset the discrete localized signaling that occurs within this system.

The idea that ROS play a central role in exercise-mediated adaptation, and that antioxidant supplementation can blunt exercise responses has received substantial attention over recent years $[65,66]$. Initially antioxidant supplementation was shown to attenuate acute exerciseinduced increases in mitochondrial and antioxidant gene expression [67], and subsequently impair endurance training mediated enhancement in mitochondrial biogenesis and insulin sensitivity in both humans and rodents [68-71]. More recently ROS have also been implicated in signaling of muscle hypertrophy in response to overloading [72-74], and mitochondrial derived signals may play a role in myogenesis $[66,75]$ and attenuation of muscle apoptosis 
[76]. However, it must be noted that other studies have reported that antioxidant supplementation does not negatively impact exercise-induced adaptations [77-81], but appear not to have any beneficial effects. These inconsistencies may reflect the use of general broadspectrum antioxidants (such as vitamin $\mathrm{C} / \mathrm{E}$ ) at a range of doses, differential training regimes, and the fact that there is likely to be redundancies as exercise stress-adaptation pathway.

ROS are produced from multiple sources during exercise [82]. Studies concerned with the role of ROS in regulating exercise-mediated adaptation have mostly utilized general antioxidants that scavenge ROS produced from all sources $[68,70,83]$. Therefore, while there is increasing evidence that ROS signaling is, at least in part, responsible for mediating exercise-induced increases in mitochondrial biogenesis within skeletal muscle, as well as antioxidant capacity and insulin sensitivity, whether this is a result of mitochondrial ROS acting as a retrograde signal (mitohormesis) or ROS from other locations is yet to be specifically investigated. Interestingly and for example, the xanthine oxidase inhibitor, allopurinol, has been shown to impair gene transcription in response to an acute bout of exercise, but it has no major effect on long-term exercise training induced increases in mitochondrial biogenesis, antioxidant defenses or GLUT4 levels [78]. Since xanthine oxidase enzymes produce ROS during exercise [67, 82], this suggests that ROS involved in exercise adaptation maybe derived from other sources.

\section{Mitochondrial membrane potential and calcium}

Mitochondrial stress is typically reflected in changes in mitochondrial membrane potential. Alterations in mitochondrial membrane potential can activate cytosolic factors and induce mitohormetic responses [84]. This may occur by altering ROS production, with increases in mitochondrial membrane potential being associated with increased ROS release, or alternatively, via calcium $\left(\mathrm{Ca}^{2+}\right)$ signaling. Mitochondrial membrane potential controls 
mitochondria $\mathrm{Ca}^{2+}$ homeostasis and alteration in mitochondrial $\mathrm{Ca}^{2+}$ flux can have a significant impact on cytosolic $\mathrm{Ca}^{2+}$ levels [85]. Inhibition of the mitochondrial respiratory chain induces ROS production but also elevates intracellular $\mathrm{Ca}^{2+}$ homeostasis and activates cytosolic CaMK/MAPK signaling pathways [86-88]. Importantly these effects can be reversed by $\mathrm{Ca}^{2+}$ chelators or partial deletion of mtDNA.

Mitochondrial $\mathrm{Ca}^{2+}$ transport is involved in regulating excitation contraction coupling in skeletal muscle [89], and skeletal muscle mitochondrial $\mathrm{Ca}^{2+}$ levels are significantly increased during and for at least 60 min following endurance exercise in humans [90]. Furthermore, the inhibition of mitochondrial $\mathrm{Ca}^{2+}$ uptake in mammalian cells with ruthenium red or Ru360 can affect activation of transcription factors that respond to exercise including PGC1 $\alpha$, JNK and NF- $\kappa B$ [91, 92]. This is consistent with studies that suggest $\mathrm{Ca}^{2+} /$ calmodulin-dependent protein kinase $(\mathrm{CaMKs})$ are activated during exercise in response to changes in intracellular $\mathrm{Ca}^{2+}$ levels and are involved in exercise-induced increases in GLUT4 [93] and mitochondrial content [94-96]. Taken together, it is possible that alterations in cytosolic $\mathrm{Ca}^{2+}$ levels mediated by the mitochondria during exercise may act as a retrograde signal to induce cellular adaptation.

\section{Mitochondrial unfolded protein response $\left(U P R^{m t}\right)$}

Perturbation to mitochondrial proteostasis leads to the accumulation of misfolded proteins in the mitochondrial matrix, which initiate a mitochondria-specific stress response that signals the transcription of nuclear-encoded mitochondrial chaperone proteins [97]. This is referred as the mitochondrial unfolded protein response $\left(\mathrm{UPR}^{\mathrm{mt}}\right)$. Direct targeting of the mitochondrial proteostasis systems, or interfering with mitochondrial matrix and electron transport chain (ETC) architecture has been shown to elicit $\mathrm{UPR}^{\mathrm{mt}}$ (as determined by transcription of 
mitochondrial chaperones and proteases), in model organisms (C. elegans and Drosophila) and mammalian cells [97-101]

Accumulated damaged or unfolded mitochondrial proteins are degraded by mitochondrial matrix proteases to small peptides which are transported out of the mitochondria. Release of these peptides may play a role in activating the major $\mathrm{UPR}^{\mathrm{mt}}$ transcription factor ATFS-1 in C. elegans [102, 103]. ATFS-1 contains a mitochondrial targeting sequence, which under normal conditions means it is shuttled to the mitochondria for degradation. However the induction of mitochondrial stress reduces mitochondrial ATFS-1 import and initiates its nuclear localization sequence to promote nuclear translocation, where it induces the UPR ${ }^{\mathrm{mt}}$ transcriptional response [103]. Unfortunately there is not a clear sequence homolog for ATFS1 in mammals, however p53 and HIF-1 show similarities in regulation since they are targeted for proteasomal degradation under non-stress conditions and have the ability to shuttle between the nucleus and mitochondria $[104,105]$. In addition to these candidates the mitochondrial single-strand DNA-binding protein 1 (SSBP1) has recently been shown to interact with HSF1 to translocate from the mitochondrial to the nucleus and promote UPR ${ }^{\mathrm{mt}}$ genes expression in response to proteotoxic stresses in mammalian cells [106]. Furthermore, HSF1 can act via the exercise sensitive transcription factor PGC1 $\alpha$ to increase mitochondrial function and elevate energy expenditure [107].

Evidence suggests that both p53 and HIF-1 play an important role in regulating skeletal muscle recovery and adaptation to repeated bouts of exercise. Skeletal muscle p53 knockout mice show no improvement in exercise performance following exercise training [108]. While the ablation of skeletal muscle HIF-1 promotes fatty acid oxidation and improves acute exercise performance, increases in skeletal muscle glucose metabolism genes following an acute bout of exercise are absent in these mice [109]. Furthermore, repeated days of exercise 
leads to muscle damage and deterioration in exercise performance that is not seen in wild type mice [109] suggesting that their exercise adaptive-recovery process is impaired. Similarly, $\mathrm{UPR}^{\mathrm{mt}}$ effector proteins, such as heat shock protein, are upregulated following exercise and appear to be involved in regulating some of the cryoprotective and thermotolerance conferred by exercise training [110]. Whether these factors are initiated by mitochondrial retrograde signaling or are solely accounted for by cytosolic mediated anterior grade nuclear signaling is yet to be determined.

$\mathrm{UPR}^{\mathrm{mt}}$ and ROS may also act as synergistic mitohormetic signals through CLK-1 (known as Mclk-1 or COQ7 in mammals), a protein residing in the mitochondria to catalyze the ubiquinone biosynthesis. CLK-1 has recently been reported to also be present in the nucleus of C. elegans and mammalian cells, where it acts to promote antioxidant responses and suppresses $\mathrm{UPR}^{\mathrm{mt}}$ gene expression [111]. Increased oxidative stress promotes CLK-1 nuclear transport which induces an antioxidant response, lowering ROS levels, but also reduces $\mathrm{UPR}^{\mathrm{mt}}$. The resulting decrease in ROS leads to CLK-1 localizing predominantly in the mitochondria. Thus, it is suggested that CLK-1 acts as a rheostat regulator for ROS homeostasis, as well as rate limiting factor for $\mathrm{UPR}^{\mathrm{mt}}$ under low stress conditions [111]. Reduced CLK-1 expression in C. elegans [112] and Mclk [113] in mice extends lifespan. It therefore appears that CLK-1 may act in the basal state to suppress ROS and UPR ${ }^{\mathrm{mt}}$, and that deletion of CLK-1 under basal conditions would promote CLK-1 independent ROS and $\mathrm{UPR}^{\mathrm{mt}}$ mediated adaption. This does not preclude the potential for CLK-1 to mediate mitochondrial initiated lifespan extending adaptations under conditions of stress. Interestingly, mild disruption of mitochondrial ETC in the muscle of Drosophila, which increases lifespan and improves muscle function, appears to be the result of ROS-mediated mitochondrial stress upregulation of UPR ${ }^{\mathrm{mt}}$ effectors [98]. This suggests that while basal ROS production may limit $\mathrm{UPR}^{\mathrm{mt}}$ signaling, this interaction is more complex and some level of 
coordination and overlap in ROS-UPR ${ }^{\mathrm{mt}}$ mitohormetic signaling is required to improve homeostatic function.

\section{Mitochondrial Metabolites}

Since the mitochondria provide energy for the cell via oxidative metabolism, changes in mitochondrial requirements or function can alter cellular energy balance and the level of metabolic intermediates. These changes are sensed by a host of protein kinases and deacetylases which in turn can coordinate nuclear gene transcription. Whether metabolic metabolites are important mitohormesis signal regulators under normal or low stress conditions is not clear, however, they could potentially fill this role during exercise. The energy sensing kinase, AMPK, is activated by a shift in AMP:ATP ratio and plays a central role not only in the regulation of acute energy metabolism during exercise but also stimulating exercise adaptation [114]. Activation of AMPK during exercise is at least partially responsible for the activation of PGC1 $\alpha$, the nuclear export of HDAC5, and the inhibition of mTOR immediately following exercise to promote increases in mitochondrial biogenesis, GLUT4 expression and protein synthesis, respectively [114, 115].

Inhibition of the mitochondrial ETC complex-1 with the anti-diabetic drug metformin, alters energy homeostasis and activates AMPK increasing skeletal muscle mitochondrial biogenesis [116] and insulin sensitivity $[117,118]$ through a pathway that partially overlaps with that of exercise training. Furthermore, chronic supplementation of mice fed a high-fat diet with the mitochondrial complex-1 inhibitor, R419, has recently been shown to enhance exercise capacity [119]. This effect was associated with an enhancement in mitochondrial ETC activity and was dependent on muscle AMPK expression, suggesting that impaired complex-1 function is sensed by AMPK which then mediates responses (via the nucleus) to enhance the function of other ETC subunits to compensate, as previously shown in C. elegans [120]. 
However, whether this pathway is involved in potential mitohormesis signaling during exercise training is unclear given that the ablation of AMPK $\alpha 2$ or PGC1 $\alpha$ has little effects on exercise-induced increases in mitochondrial biogenesis and glucose metabolism markers [121-123]. Unfortunately, interpretations of results from AMPKa2 knockout mice are complicated by compensatory increases in AMPK $\alpha 1$ activity [121].

Exercise is associated with a large increase in intramuscular levels of the mitochondrial metabolism intermediate $\mathrm{NAD}^{+}$[124], which acts as a substrate for the deacetylating sirtuin enzymes. While exercise increases sirtuin activity and modulation of SIRT1 expression in the basal state alters mitochondrial level and function [124-126], SIRT1 appears to play little role in exercise-training mediated adaptations [127]. However, increasing $\mathrm{NAD}^{+}$levels through the inhibition of the $\mathrm{NAD}^{+}$consuming enzymes poly(ADP-ribose) polymerase-1 (PARP-1) enhances mitochondrial ETC function and exercise capacity in mice [128] which may be mediated through NNMT $[129,130]$. This suggests that $\mathrm{NAD}^{+}$could act as a mitochondrialderived signaling intermediate to induced mitochondrial biogenesis during exercise training, however the targets of $\mathrm{NAD}^{+}$during exercise are yet to be established.

\section{Mitokines}

Currently only two peptides, humanin and mitochondrial open reading frame of the $12 \mathrm{~S}$ rRNA-c (MOTS-c), have been found to have a short open reading frame (sORF) mapping to mitochondrial DNA [131-133]. While it is not clear whether these peptides are translated in the mitochondria or cytoplasm (or both) in vivo, given the increasingly realization of the complexity of the mitochondrial transcriptome [134], they potentially represent mitochondrial-derived peptides (mitokines). Importantly, they meet the requirements of an mitohormetic signal transducer as both have been shown to not only elicit mitochondrial adaptations, but promote systemic responses which protect against the deleterious effects of 
numerous cryostressors [131] and metabolic disturbances [132, 135]. Physiological perturbations that induce transcription and systemic secretion of these peptides have not been well established, but inhibition of mitochondrial translation appears to play a role. Whether mitokine levels are altered in response to exercise stress is not known but is intriguing question, particularly given their propensity to protect against metabolic disease and the relatively recent evidence that exercise-stimulated myokines have a similar effect [136]. Furthermore, mitochondrial ribosomal protein L2 gene expression is upregulated following acute exercise in humans [137], suggesting that mitochondrial translation is altered by exercise.

\section{Mitochondrial dysfunction models and exercise training}

In the previous sections we have discussed potential emitters and transcription factors through which the mitochondrial may translate exercise-stress to evoke a nuclear transcription response and initiate exercise adaptations (Figure 2). However, there is little causative evidence directly linking mitohormesis to exercise responses. A common approach in examining mitohormesis signals is determining whether normal hormetic response can be achieved in cells or lower organisms with mutated or ablated mtDNA. While applying this approach to in vivo exercise situation is more difficult due to the requirement of mtDNA for normal cellular function and the potential for mtDNA mutation to elicit a mitohormetic response itself. However, several groups have assessed the effectiveness of exercise training in mouse models of mitochondrial dysfunction.

Mice with defective mtDNA polymerase function (mtDNA mutator mouse) have impaired mitochondrial function, and their muscles are weaker and more susceptible to fatigue [138]. Despite reduced mitochondrial ATP production, under the basal sate these mice have lower mitochondrial biogenesis signaling (AMPK and PGC1 $\alpha$ ) suggesting that a defect in mtDNA 
prevents compensatory mitochondrial expansion [138]. Overexpression of PGC1 $\alpha$ in the muscle of these mice restores mitochondrial function and improves muscle function [139]. Similar improvements in mitochondrial and muscle function are seen when PGC1 $\alpha$ is overexpressed in the muscle of mice lacking the COX10 gene [140], which is a cytochrome oxidase (COX) assembly factor. This suggests that in these models the ability of the mitochondria to effectively emit a signal to activate mitochondrial biogenesis transcription factors is impaired. Surprisingly, however, endurance exercise training was also able to induce increases in PGC1 $\alpha$ in both these mouse models and this was associated with improved mitochondrial function and the restoration of normal endurance capacity [141, 142]. Unfortunately, an exercise-trained control group was not included in these studies making it difficult to assess whether the training response in these mouse models was to the same extent as would be seen in wild type mice. Regardless, this suggests that the exercise stimulus was sufficient to overcome impairments in basal mitohormesis defects. Whether this is attributed to non-mitohormesis activation of exercise adaptation, or that the defects in the mitochondria were insufficient to prevent mitohormesis during exercise requires further investigation. Similar to the mtDNA mutator and COX10 mice, Mclk+/- mice, which have impaired mitochondrial ETC function, have reduced oxidative capacity $[143,144]$. When exposed to a voluntary running wheel Mclk+/- mice complete substantially more work than wild type mice [144]. However, this increased activity is insufficient to rescue their impairment in exercise capacity meaning that they are not responsive to exercise training. This may be the result of reduced mitohormetic induced transcriptional response to exercise.

\section{Conclusion}

Taken together, there is evidence that mitohormesis is an important signaling pathway that promotes systemic adaptations which increase cellular tolerance to future stressors. A number of transcription factors are known to be activated in response to exercise and promote both 
mitochondrial and cell- and system-wide adaptations. Furthermore, these transcription factors appear to be sensitive to potential mitochondrial retrograde signals that are emitted during exercise, with mitochondrial ROS being a primary candidate for initiating mitohormetic response. Causative evidence linking mitohormesis to exercise adaptive response is limited, however, given that altering potential mitohormetic emitters in the basal state or during exercise-training can affect markers of exercise adaptations it appears highly likely that mitohormetic signaling plays a central role in regulating the adaptive response to exercise.

\section{Acknowledgements}

The authors thank all former and previous laboratory members that have contributed to this review. Work and personnel that contributed to this were supported by the research program of the Jena Centre for Systems Biology of Aging (JenAge) funded by the German Ministry for Education and Research (Bundesministerium für Bildung und Forschung / BMBF 0315581), the German Research Association (Deutsche Forschungsgemeinschaft, DFG) Research Training Group 1715 Molecular Signatures of Adaptive Stress Responses, Jena, Germany, the Swiss National Science Foundation (Schweizerischer Nationalfonds, SNF 31003A_156031), the Novartis Foundation for Medical-Biological Research $(14 \mathrm{C} 149)$ and the European Foundation for the Study of Diabetes (EFSD/Lilly Research Fellowship Programme).

The authors declare no conflict of interest. 


\section{References}

1. Egan, B. and J.R. Zierath, Exercise metabolism and the molecular regulation of skeletal muscle adaptation. Cell Metab, 2013. 17(2): p. 162-84.

2. Warburton, D.E., C.W. Nicol, and S.S. Bredin, Health benefits of physical activity: the evidence. Can Med Ass J (CMAJ), 2006. 174(6): p. 801-9.

3. Selye, H., A syndrome produced by diverse nocuous agents. Nature, 1936. 138(3479): p. 32 .

4. Selye, H. and C. Fortier, Adaptive reactions to stress. Res Publ Assoc Res Nerv Ment Dis, 1949. 29: p. 3-18.

5. Southam, C.M. and J. Ehrlich, Effects of extract of western red-cedar heartwood on certain wood-decaying fungi in culture. Phytopathology, 1943. 33: p. 517-24.

6. von Hohenheim (a.k.a. Paracelsus), P.T.A.B., Labyrintus und Irrgang der vermeinten Artzet. Item, Siben defensiones, oder Schirmreden., ed. S. Apiarium. 1574, Basel: Petrus Perna.

7. Schulz, H.P.F., Zur Lehre von der Arzneiwirkung. Virchows Archiv für pathologische Anatomie und Physiologie und für klinische Medizin, 1887. 108: p. 423-445.

8. Hueppe, F., Principles of Bacteriology Translated from the German by Jordon EO, translator. 1896, The Open Court Publishing Company: Chicago.

9. Holloszy, J.O. and E.F. Coyle, Adaptations of skeletal muscle to endurance exercise and their metabolic consequences. J Appl Physiol Respir Environ Exerc Physiol, 1984. 56(4): p. 831-8.

10. Hawley, J.A. and J.P. Morton, Ramping up the signal: promoting endurance training adaptation in skeletal muscle by nutritional manipulation. Clin Exp Pharmacol Physiol, 2014. 41(8): p. 608-13.

11. Wyndham, C.H., The physiology of exercise under heat stress. Annu Rev Physiol, 1973. 35: p. 193-220. 
12. Snyder, A.C., Overtraining and glycogen depletion hypothesis. Med Sci Sports Exerc, 1998. 30(7): p. 1146-50.

13. Tapia, P.C., Sublethal mitochondrial stress with an attendant stoichiometric augmentation of reactive oxygen species may precipitate many of the beneficial alterations in cellular physiology produced by caloric restriction, intermittent fasting, exercise and dietary phytonutrients: 'Mitohormesis' for health and vitality. Med Hypotheses, 2006. 66(4): p. 832-43.

14. Ristow, M. and K. Zarse, How increased oxidative stress promotes longevity and metabolic health: The concept of mitochondrial hormesis (mitohormesis). Exp Gerontol, 2010. 45(6): p. 410-8.

15. Yun, J. and T. Finkel, Mitohormesis. Cell Metab, 2014.

16. Schulz, T.J., et al., Glucose restriction extends Caenorhabditis elegans life span by inducing mitochondrial respiration and increasing oxidative stress. Cell Metab, 2007. 6(4): p. 280-293.

17. Anderson, S., et al., Sequence and organization of the human mitochondrial genome. Nature, 1981. 290(5806): p. 457-65.

18. Parikh, V.S., et al., The mitochondrial genotype can influence nuclear gene expression in yeast. Science, 1987. 235(4788): p. 576-80.

19. Epstein, C.B., et al., Genome-wide responses to mitochondrial dysfunction. Mol Biol Cell, 2001. 12(2): p. 297-308.

20. Liu, Z. and R.A. Butow, Mitochondrial retrograde signaling. Annu Rev Genet, 2006. 40: p. $159-85$.

21. Liao, X.S., et al., Intramitochondrial functions regulate nonmitochondrial citrate synthase (CIT2) expression in Saccharomyces cerevisiae. Mol Cell Biol, 1991. 11(1): p. $38-46$. 
22. Jia, Y., et al., A basic helix-loop-helix-leucine zipper transcription complex in yeast functions in a signaling pathway from mitochondria to the nucleus. Mol Cell Biol, 1997. 17(3): p. 1110-7.

23. Liao, X. and R.A. Butow, RTG1 and RTG2: two yeast genes required for a novel path of communication from mitochondria to the nucleus. Cell, 1993. 72(1): p. 61-71.

24. Butow, R.A. and N.G. Avadhani, Mitochondrial signaling: the retrograde response. Mol Cell, 2004. 14(1): p. 1-15.

25. Liu, Z., et al., RTG-dependent mitochondria to nucleus signaling is negatively regulated by the seven WD-repeat protein Lst8p. EMBO J, 2001. 20(24): p. 7209-19.

26. Chae, S., et al., A systems approach for decoding mitochondrial retrograde signaling pathways. Sci Signal, 2013. 6(264): p. rs4.

27. Handschin, C. and B.M. Spiegelman, The role of exercise and PGClalpha in inflammation and chronic disease. Nature, 2008. 454(7203): p. 463-9.

28. Hawley, J.A., et al., Integrative biology of exercise. Cell, 2014. 159(4): p. 738-49.

29. Ji, L.L., Modulation of skeletal muscle antioxidant defense by exercise: Role of redox signaling. Free Radic Biol Med, 2008. 44(2): p. 142-52.

30. Kojda, G. and D. Harrison, Interactions between NO and reactive oxygen species: pathophysiological importance in atherosclerosis, hypertension, diabetes and heart failure. Cardiovasc Res, 1999. 43(3): p. 562-71.

31. Houstis, N., E.D. Rosen, and E.S. Lander, Reactive oxygen species have a causal role in multiple forms of insulin resistance. Nature, 2006. 440(7086): p. 944-8.

32. Uttara, B., et al., Oxidative stress and neurodegenerative diseases: a review of upstream and downstream antioxidant therapeutic options. Curr Neuropharmacol, 2009. 7(1): p. 65-74. 
33. Wiseman, H. and B. Halliwell, Damage to DNA by reactive oxygen and nitrogen species: role in inflammatory disease and progression to cancer. Biochem J, 1996. 313 ( Pt 1): p. 17-29.

34. Harman, D., Aging: a theory based on free radical and radiation chemistry. J Gerontol, 1956. 11(3): p. 298-300.

35. Phillips, J.P., et al., Null mutation of copper/zinc superoxide dismutase in Drosophila confers hypersensitivity to paraquat and reduced longevity. Proc Natl Acad Sci U S A, 1989. 86(8): p. 2761-5.

36. Orr, W.C. and R.S. Sohal, Extension of life-span by overexpression of superoxide dismutase and catalase in Drosophila melanogaster. Science, 1994. 263(5150): p. 1128-30.

37. Melov, S., et al., Extension of life-span with superoxide dismutase/catalase mimetics. Science, 2000. 289(5484): p. 1567-9.

38. Moskovitz, J., et al., Methionine sulfoxide reductase (MsrA) is a regulator of antioxidant defense and lifespan in mammals. Proc Natl Acad Sci U S A, 2001. 98(23): p. 12920-5.

39. Zou, S., et al., Comparative approaches to facilitate the discovery of prolongevity interventions: effects of tocopherols on lifespan of three invertebrate species. Mech Ageing Dev, 2007. 128(2): p. 222-6.

40. Ristow, M., Unraveling the truth about antioxidants: mitohormesis explains ROSinduced health benefits. Nat Med, 2014. 20(7): p. 709-11.

41. Zarse, K., et al., Impaired insulin/IGF1-signaling extends life span by promoting mitochondrial L-proline catabolism to induce a transient ROS signal. Cell Metab, 2012. 15(4): p. 451-465.

42. Sena, L.A. and N.S. Chandel, Physiological roles of mitochondrial reactive oxygen species. Mol Cell, 2012. 48(2): p. 158-67. 
43. Greenberg, E.R., et al., A clinical trial of antioxidant vitamins to prevent colorectal adenoma. Polyp Prevention Study Group. N Engl J Med, 1994. 331(3): p. 141-7.

44. Rautalahti, M.T., et al., The effects of supplementation with alpha-tocopherol and beta-carotene on the incidence and mortality of carcinoma of the pancreas in a randomized, controlled trial. Cancer, 1999. 86(1): p. 37-42.

45. Virtamo, J., et al., Effects of supplemental alpha-tocopherol and beta-carotene on urinary tract cancer: incidence and mortality in a controlled trial (Finland). Cancer Causes Control, 2000. 11(10): p. 933-9.

46. Zureik, M., et al., Effects of long-term daily low-dose supplementation with antioxidant vitamins and minerals on structure and function of large arteries. Arterioscler Thromb Vasc Biol, 2004. 24(8): p. 1485-91.

47. Czernichow, S., et al., Effect of supplementation with antioxidants upon long-term risk of hypertension in the SU.VI.MAX study: association with plasma antioxidant levels. $\mathrm{J}$ Hypertens, 2005. 23(11): p. 2013-8.

48. Czernichow, S., et al., Antioxidant supplementation does not affect fasting plasma glucose in the Supplementation with Antioxidant Vitamins and Minerals (SU.VI.MAX) study in France: association with dietary intake and plasma concentrations. Am $\mathbf{J}$ Clin Nutr, 2006. 84(2): p. 395-9.

49. Sesso, H.D., et al., Vitamins $E$ and $C$ in the prevention of cardiovascular disease in men: the Physicians' Health Study II randomized controlled trial. JAMA, 2008. 300(18): p. 2123-33.

50. Albanes, D., et al., Alpha-Tocopherol and beta-carotene supplements and lung cancer incidence in the alpha-tocopherol, beta-carotene cancer prevention study: effects of base-line characteristics and study compliance. J Natl Cancer Inst, 1996. 88(21): p. 1560-70. 
51. Omenn, G.S., et al., Effects of a combination of beta carotene and vitamin A on lung cancer and cardiovascular disease. N Engl J Med, 1996. 334(18): p. 1150-5.

52. Vivekananthan, D.P., et al., Use of antioxidant vitamins for the prevention of cardiovascular disease: meta-analysis of randomised trials. Lancet, 2003. 361(9374): p. 2017-23.

53. Lonn, E., et al., Effects of long-term vitamin E supplementation on cardiovascular events and cancer: a randomized controlled trial. JAMA, 2005. 293(11): p. 1338-47.

54. Bjelakovic, G., et al., Mortality in randomized trials of antioxidant supplements for primary and secondary prevention: systematic review and meta-analysis. JAMA, 2007. 297(8): p. 842-57.

55. Fortmann, S.P., et al., Vitamin and mineral supplements in the primary prevention of cardiovascular disease and cancer: An updated systematic evidence review for the U.S. Preventive Services Task Force. Ann Intern Med, 2013. 159(12): p. 824-34.

56. Bjelakovic, G., D. Nikolova, and C. Gluud, Antioxidant supplements and mortality. Curr Opin Clin Nutr Metab Care, 2014. 17(1): p. 40-4.

57. Powers, S.K., et al., Reactive oxygen species are signaling molecules for skeletal muscle adaptation. Exp Physiol, 2010. 95(1): p. 1-9.

58. Tiganis, T., Reactive oxygen species and insulin resistance: the good, the bad and the ugly. Trends Pharmacol Sci, 2011. 32(2): p. 82-9.

59. Faraci, F.M., Hydrogen peroxide: watery fuel for change in vascular biology. Arterioscler Thromb Vasc Biol, 2006. 26(9): p. 1931-3.

60. Thannickal, V.J. and B.L. Fanburg, Reactive oxygen species in cell signaling. Am J Physiol Lung Cell Mol Physiol, 2000. 279(6): p. L1005-28.

61. Zuo, L., et al., Biological and physiological role of ROS-the good, the bad and the ugly. Acta Physiol (Oxf), 2015. 
62. Nojima, A., et al., Haploinsufficiency of akt1 prolongs the lifespan of mice. PLoS One, 2013. 8(7): p. e69178.

63. Katic, M., et al., Mitochondrial gene expression and increased oxidative metabolism: role in increased lifespan of fat-specific insulin receptor knock-out mice. Aging Cell, 2007. 6(6): p. 827-39.

64. Brown-Borg, H.M., W.T. Johnson, and S.G. Rakoczy, Expression of oxidative phosphorylation components in mitochondria of long-living Ames dwarf mice. Age (Dordr), 2012. 34(1): p. 43-57.

65. Gomez-Cabrera, M.C., et al., Redox modulation of mitochondriogenesis in exercise. Does antioxidant supplementation blunt the benefits of exercise training? Free Radic Biol Med, 2015.

66. Barbieri, E., et al., Mitohormesis in muscle cells: a morphological, molecular, and proteomic approach. Muscles Ligaments Tendons J, 2013. 3(4): p. 254-66.

67. Gomez-Cabrera, M.C., et al., Decreasing xanthine oxidase-mediated oxidative stress prevents useful cellular adaptations to exercise in rats. J Physiol, 2005. 567(Pt 1): p. 113-20.

68. Gomez-Cabrera, M.C., et al., Oral administration of vitamin $C$ decreases muscle mitochondrial biogenesis and hampers training-induced adaptations in endurance performance. Am J Clin Nutr, 2008. 87(1): p. 142-9.

69. Strobel, N.A., et al., Antioxidant supplementation reduces skeletal muscle mitochondrial biogenesis. Med Sci Sports Exerc, 2011. 43(6): p. 1017-24.

70. Paulsen, G., et al., Vitamin $C$ and E supplementation hampers cellular adaptation to endurance training in humans: a double-blind randomized controlled trial. J Physiol, 2014. 592(8): p. 1887-1901.

71. Ristow, M., et al., Antioxidants prevent health-promoting effects of physical exercise in humans. Proc Nat Acad Sci, 2009. 106(21): p. 8665-70. 
72. Paulsen, G., et al., Vitamin $C$ and $E$ supplementation alters protein signalling after a strength training session, but not muscle growth during 10 weeks of training. $\mathrm{J}$ Physiol, 2014. 592(Pt 24): p. 5391-408.

73. Makanae, Y., et al., Vitamin C administration attenuates overload-induced skeletal muscle hypertrophy in rats. Acta Physiol (Oxf), 2013. 208(1): p. 57-65.

74. Ito, N., et al., Activation of calcium signaling through Trpv1 by nNOS and peroxynitrite as a key trigger of skeletal muscle hypertrophy. Nat Med, 2013. 19(1): p. 101-6.

75. Barbieri, E., et al., Morphofunctional and biochemical approaches for studying sitochondrial changes during myoblasts differentiation. J Aging Res, 2011. 2011: p. 845379.

76. Adhihetty, P.J., M.F. O'Leary, and D.A. Hood, Mitochondria in skeletal muscle: adaptable rheostats of apoptotic susceptibility. Exerc Sport Sci Rev, 2008. 36(3): p. $116-21$.

77. Wadley, G.D. and G.K. McConell, High dose antioxidant vitamin C supplementation does not prevent acute exercise-induced increases in markers of skeletal muscle mitochondrial biogenesis in rats. J Appl Physiol, 2010. 108(6): p. 1719-26.

78. Wadley, G.D., et al., Xanthine oxidase inhibition attenuates skeletal muscle signaling following acute exercise but does not impair mitochondrial adaptations to endurance training. Am J Physiol Endocrinol Metab, 2013. 304(8): p. E853-62.

79. Higashida, K., et al., Normal adaptations to exercise despite protection against oxidative stress. Am J Physiol Endocrinol Metab, 2011. 301(5): p. E779-84.

80. Yfanti, C., et al., Antioxidant supplementation does not alter endurance training adaptation. Med Sci Sports Exerc, 2010. 42(7): p. 1388-95. 
81. Yfanti, C., et al., Effect of antioxidant supplementation on insulin sensitivity in response to endurance exercise training. Am J Physiol Endocrinol Metab, 2011. 300(5): p. E761-70.

82. Powers, S.K. and M.J. Jackson, Exercise-induced oxidative stress: cellular mechanisms and impact on muscle force production. Physiol Rev, 2008. 88(4): p. 1243-76.

83. Ristow, M., et al., Frataxin activates mitochondrial energy conversion and oxidative phosphorylation. Proc Natl Acad Sci U S A, 2000. 97(22): p. 12239-43.

84. Jin, S.M., et al., Mitochondrial membrane potential regulates PINK1 import and proteolytic destabilization by PARL. J Cell Biol, 2010. 191(5): p. 933-42.

85. Nicholls, D. and K. Akerman, Mitochondrial calcium transport. Biochim Biophys Acta, 1982. 683(1): p. 57-88.

86. Arnould, T., et al., CREB activation induced by mitochondrial dysfunction is a new signaling pathway that impairs cell proliferation. EMBO J, 2002. 21(1-2): p. 53-63.

87. Luo, Y., J.D. Bond, and V.M. Ingram, Compromised mitochondrial function leads to increased cytosolic calcium and to activation of MAP kinases. Proc Natl Acad Sci U S A, 1997. 94(18): p. 9705-10.

88. Biswas, G., et al., Retrograde Ca2+ signaling in C2C12 skeletal myocytes in response to mitochondrial genetic and metabolic stress: a novel mode of inter-organelle crosstalk. EMBO J, 1999. 18(3): p. 522-33.

89. Yi, J., et al., Mitochondrial calcium uptake regulates rapid calcium transients in skeletal muscle during excitation-contraction (E-C) coupling. J Biol Chem, 2011. 286(37): p. 32436-43.

90. Madsen, K., P. Ertbjerg, and P.K. Pedersen, Calcium content and respiratory control index of isolated skeletal muscle mitochondria: effects of different isolation media. Anal Biochem, 1996. 237(1): p. 37-41. 
91. Lim, J.H., et al., Coupling mitochondrial dysfunction to endoplasmic reticulum stress response: a molecular mechanism leading to hepatic insulin resistance. Cell Signal, 2009. 21(1): p. 169-77.

92. Waris, G., et al., Hepatitis $C$ virus NS5A and subgenomic replicon activate NFkappaB via tyrosine phosphorylation of IkappaBalpha and its degradation by calpain protease. J Biol Chem, 2003. 278(42): p. 40778-87.

93. Smith, J.A., et al., Exercise and CaMK activation both increase the binding of MEF2A to the Glut4 promoter in skeletal muscle in vivo. Am J Physiol Endocrinol Metab, 2007. 292(2): p. E413-20.

94. Wu, H., et al., Regulation of mitochondrial biogenesis in skeletal muscle by CaMK. Science, 2002. 296(5566): p. 349-52.

95. Wright, D.C., et al., Calcium induces increases in peroxisome proliferator-activated receptor gamma coactivator-1alpha and mitochondrial biogenesis by a pathway leading to p38 mitogen-activated protein kinase activation. J Biol Chem, 2007. 282(26): p. 18793-9.

96. Wright, D.C., et al., Exercise-induced mitochondrial biogenesis begins before the increase in muscle PGC-1\{alpha\} expression. J Biol Chem, 2007. 282(1): p. 194-9.

97. Jovaisaite, V. and J. Auwerx, The mitochondrial unfolded protein responsesynchronizing genomes. Curr Opin Cell Biol, 2015. 33: p. 74-81.

98. Owusu-Ansah, E., W. Song, and N. Perrimon, Muscle mitohormesis promotes longevity via systemic repression of insulin signaling. Cell, 2013. 155(3): p. 699-712.

99. Durieux, J., S. Wolff, and A. Dillin, The cell-non-autonomous nature of electron transport chain-mediated longevity. Cell, 2011. 144(1): p. 79-91.

100. Yoneda, T., et al., Compartment-specific perturbation of protein handling activates genes encoding mitochondrial chaperones. J Cell Sci, 2004. 117(Pt 18): p. 4055-66. 
101. Runkel, E.D., et al., Surveillance-activated defenses block the ROS-induced mitochondrial unfolded protein response. PLoS Genet, 2013. 9(3): p. e1003346.

102. Haynes, C.M. and D. Ron, The mitochondrial UPR - protecting organelle protein homeostasis. J Cell Sci, 2010. 123(Pt 22): p. 3849-55.

103. Nargund, A.M., et al., Mitochondrial import efficiency of ATFS-1 regulates mitochondrial UPR activation. Science, 2012. 337(6094): p. 587-90.

104. Vaseva, A.V. and U.M. Moll, The mitochondrial p53 pathway. Biochim Biophys Acta, 2009. 1787(5): p. 414-20.

105. Boutin, A.T., et al., Epidermal sensing of oxygen is essential for systemic hypoxic response. Cell, 2008. 133(2): p. 223-34.

106. Tan, K., et al., Mitochondrial SSBP1 protects cells from proteotoxic stresses by potentiating stress-induced HSF1 transcriptional activity. Nat Commun, 2015. 6: p. 6580.

107. Ma, X., et al., Celastrol Protects against Obesity and Metabolic Dysfunction through Activation of a HSF 1-PGClalpha Transcriptional Axis. Cell Metab, 2015. 22(4): p. 695-708.

108. Park, J.Y., et al., p53 improves aerobic exercise capacity and augments skeletal muscle mitochondrial DNA content. Circ Res, 2009. 105(7): p. 705-12, 11 p following 712.

109. Mason, S.D., et al., Loss of skeletal muscle HIF-1alpha results in altered exercise endurance. PLoS Biol, 2004. 2(10): p. e288.

110. Kregel, K.C., Heat shock proteins: modifying factors in physiological stress responses and acquired thermotolerance. J Appl Physiol (1985), 2002. 92(5): p. 2177-86.

111. Monaghan, R.M., et al., A nuclear role for the respiratory enzyme CLK-1 in regulating mitochondrial stress responses and longevity. Nat Cell Biol, 2015. 17(6): p. 782-92. 
112. Wong, A., P. Boutis, and S. Hekimi, Mutations in the clk-1 gene of Caenorhabditis elegans affect developmental and behavioral timing. Genetics, 1995. 139(3): p. 124759.

113. Liu, X., et al., Evolutionary conservation of the clk-1-dependent mechanism of longevity: loss of mclk1 increases cellular fitness and lifespan in mice. Genes Dev, 2005. 19(20): p. 2424-34.

114. Richter, E.A. and N.B. Ruderman, $A M P K$ and the biochemistry of exercise: implications for human health and disease. Biochem J, 2009. 418(2): p. 261-75.

115. Richter, E.A. and M. Hargreaves, Exercise, GLUT4, and skeletal muscle glucose uptake. Physiol Rev, 2013.93(3): p. 993-1017.

116. Suwa, M., et al., Metformin increases the PGC-1\{alpha\} protein and oxidative enzyme activities possibly via AMPK phosphorylation in skeletal muscle in vivo. J Appl Physiol, 2006.

117. Malin, S.K., et al., Independent and combined effects of exercise training and metformin on insulin sensitivity in individuals with prediabetes. Diabetes Care, 2012. 35(1): p. 131-6.

118. Al-Khalili, L., et al., Enhanced insulin-stimulated glycogen synthesis in response to insulin, metformin or rosiglitazone is associated with increased mRNA expression of GLUT4 and peroxisomal proliferator activator receptor gamma co-activator 1. Diabetologia, 2005. 48(6): p. 1173-9.

119. Marcinko, K., et al., The AMPK activator $R 419$ improves exercise capacity and skeletal muscle insulin sensitivity in obese mice. Molecular Metabolism, 2015. 4(9): p. 643-651.

120. Schmeisser, S., et al., Neuronal ROS signaling rather than AMPK/Sirtuin-mediated energy sensing links dietary restriction to lifespan extension. Molecular Metabolism, 2013. 2: p. 92-102. 
121. Jorgensen, S.B., et al., Role of AMPKalpha2 in basal, training-, and AICAR-induced GLUT4, hexokinase II, and mitochondrial protein expression in mouse muscle. Am J Physiol Endocrinol Metab, 2007. 292(1): p. E331-9.

122. Holmes, B.F., et al., AMP kinase is not required for the GLUT4 response to exercise and denervation in skeletal muscle. Am J Physiol Endocrinol Metab, 2004. 287(4): p. E739-43.

123. Leick, L., et al., PGC-lalpha is not mandatory for exercise- and training-induced adaptive gene responses in mouse skeletal muscle. Am J Physiol Endocrinol Metab, 2008. 294(2): p. E463-74.

124. White, A.T. and S. Schenk, NAD+/NADH and skeletal muscle mitochondrial adaptations to exercise. Am J Physiol Endocrinol Metab, 2012. 303(3): p. E308-21.

125. Price, N.L., et al., SIRT1 is required for AMPK activation and the beneficial effects of resveratrol on mitochondrial function. Cell Metab, 2012. 15(5): p. 675-90.

126. Menzies, K.J., et al., Sirtuin 1-mediated effects of exercise and resveratrol on mitochondrial biogenesis. J Biol Chem, 2013. 288(10): p. 6968-79.

127. Philp, A., et al., Sirtuin 1 (SIRT1) deacetylase activity is not required for mitochondrial biogenesis or peroxisome proliferator-activated receptor-gamma coactivator-1alpha (PGC-1alpha) deacetylation following endurance exercise. J Biol Chem, 2011. 286(35): p. 30561-70.

128. Pirinen, E., et al., Pharmacological Inhibition of poly(ADP-ribose) polymerases improves fitness and mitochondrial function in skeletal muscle. Cell Metab, 2014. 19(6): p. 1034-41.

129. Schmeisser, K., et al., Role of sirtuins in lifespan regulation is linked to methylation of nicotinamide. Nat Chem Biol, 2013. 9: p. 693-700.

130. Liu, K.Y., et al., Nicotinamide N-Methyltransferase increases complex I activity IN SH-SY5Y cells via SIRTUIN-3. Biochem Biophys Res Commun, 2015. 
131. Lee, C., K. Yen, and P. Cohen, Humanin: a harbinger of mitochondrial-derived peptides? Trends Endocrinol Metab, 2013. 24(5): p. 222-8.

132. Lee, C., et al., The mitochondrial-derived peptide MOTS-c promotes metabolic homeostasis and reduces obesity and insulin resistance. Cell Metab, 2015. 21(3): p. $443-54$

133. Guo, B., et al., Humanin peptide suppresses apoptosis by interfering with Bax activation. Nature, 2003. 423(6938): p. 456-61.

134. Mercer, T.R., et al., The human mitochondrial transcriptome. Cell, 2011. 146(4): p. $645-58$.

135. Muzumdar, R.H., et al., Humanin: a novel central regulator of peripheral insulin action. PLoS One, 2009. 4(7): p. e6334.

136. Bostrom, P., et al., A PGCl-alpha-dependent myokine that drives brown-fat-like development of white fat and thermogenesis. Nature, 2012.

137. Mahoney, D.J., et al., Analysis of global mRNA expression in human skeletal muscle during recovery from endurance exercise. FASEB J, 2005. 19(11): p. 1498-500.

138. Yamada, T., et al., Impaired mitochondrial respiration and decreased fatigue resistance followed by severe muscle weakness in skeletal muscle of mitochondrial DNA mutator mice. J Physiol, 2012. 590(Pt 23): p. 6187-97.

139. Dillon, L.M., et al., Increased mitochondrial biogenesis in muscle improves aging phenotypes in the mtDNA mutator mouse. Hum Mol Genet, 2012. 21(10): p. 2288-97.

140. Wenz, T., et al., Activation of the PPAR/PGC-1alpha pathway prevents a bioenergetic deficit and effectively improves a mitochondrial myopathy phenotype. Cell Metab, 2008. 8(3): p. 249-56.

141. Wenz, T., et al., Endurance exercise is protective for mice with mitochondrial myopathy. J Appl Physiol (1985), 2009. 106(5): p. 1712-9. 
142. Safdar, A., et al., Endurance exercise rescues progeroid aging and induces systemic mitochondrial rejuvenation in mtDNA mutator mice. Proc Natl Acad Sci U S A, 2011. 108(10): p. 4135-40.

143. Lapointe, J. and S. Hekimi, Early mitochondrial dysfunction in long-lived Mclk1+/mice. J Biol Chem, 2008. 283(38): p. 26217-27.

144. Lapointe, J., et al., Compensatory elevation of voluntary activity in mouse mutants with impaired mitochondrial energy metabolism. Physiol Rep, 2014. 2(11). 


\section{Figure legends}

Figure 1. Hormetic and theoretical liner response to stress. Theoretically the exposure of a cell to a stressor results in detrimental effects in cellular function which are proportional to the level of exposure (liner response). Hormesis refers to a response where a low exposure to a stressor results in beneficial adaptions which improve the function of the cell, but once a threshold level of exposure is surpassed further exposure leads to cellular damage (hormetic response). Potentially no or very little exposure to stressors may also be a disadvantage to the homeostatic function of the cell.

Figure 2. Potential mitohormetic response to exercise. Exercise induces mitochondrial stress and as a result signals are sent from the mitochondrion to the nucleus to induce mitochondrial specific, cell- and potentially system-wide adaptive responses which protect the cell against subsequent stress (mitohormesis). Potential signals emitted by mitochondria during or immediately following exercise may include, but are not limited to, changes in ROS, $\mathrm{Ca}^{2+}, \mathrm{UPR}^{\mathrm{mt}}$, metabolic metabolites and mitokine levels. These may act directly to initiate a transcriptional response in the nucleus or via signaling intermediates such as protein kinases (not depicted in this figure but may include AMPK, mTOR, CaMK's and MAPK's) and exercise-sensitive transcription factors. AMPK, 5' adenosine monophosphate-activated protein kinase; CaMK, $\mathrm{Ca}^{2+l}$ calmodulin-dependent; HIF-1, hypoxia inducible factor; MAPK, mitogen-activated protein kinases; mTOR, mechanistic target of rapamycin; mtTFA, mitochondrial transcription factor $\mathrm{A}$; $\mathrm{NF}-\kappa \mathrm{B}$, nuclear factor kappa-light-chain-enhancer of activated $\mathrm{B}$ cells; PGC1 $\alpha$, peroxisome proliferator-activated receptor gamma coactivator $1 \alpha$; ROS, reactive oxygen species. 
Figure 1

- Linier response

- Hormetic response

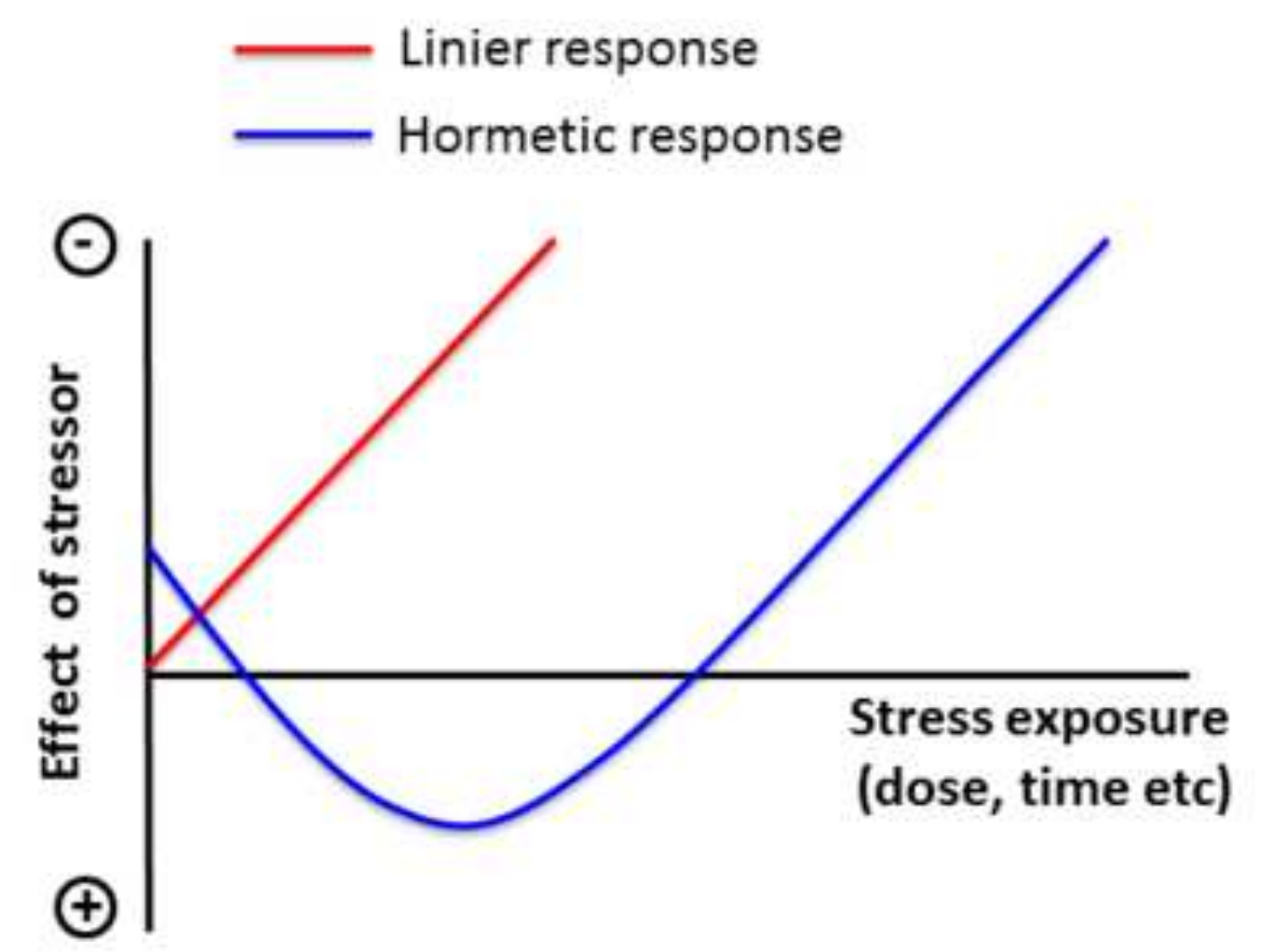




\section{Figure 2}

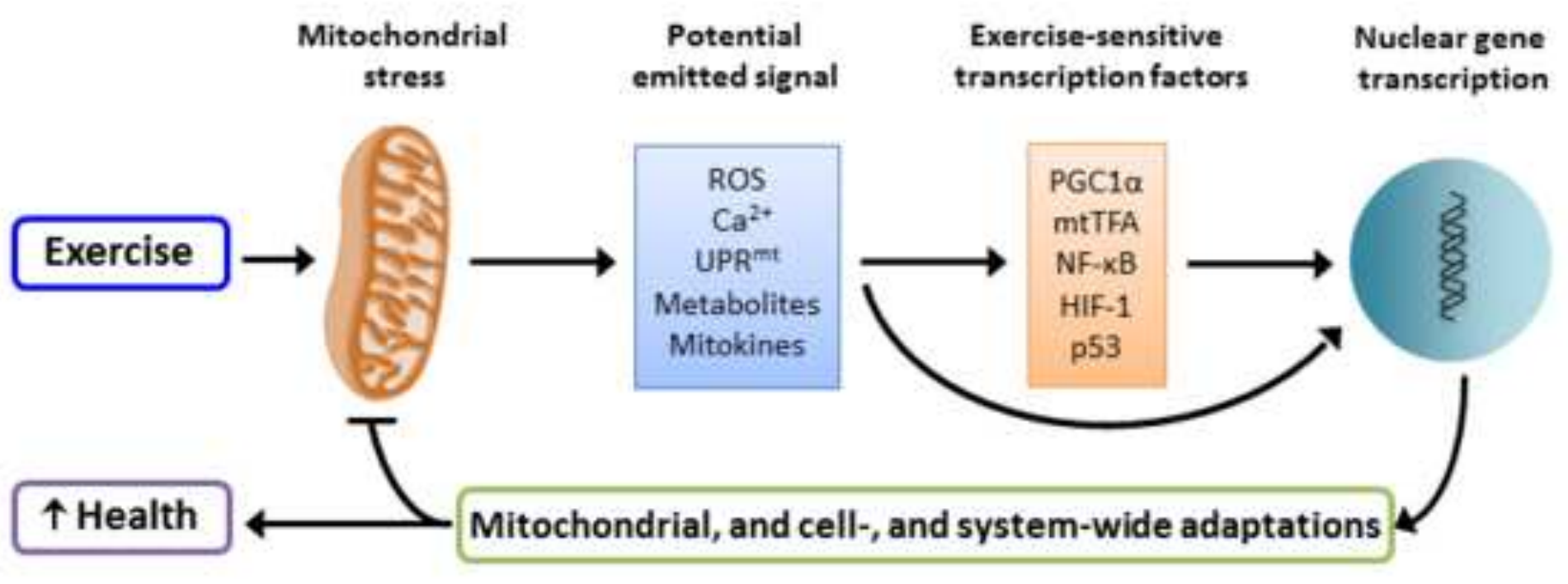

